\title{
Obesidade e síndrome dos ovários policísticos: vínculo fisiopatológico e impacto no fenótipo das pacientes
}

\author{
Obesity and polycystic ovary syndrome: \\ pathophysiological link and impact on patients' phenotype
}

Lenora Maria C. S. M. Leão*

\begin{abstract}
Resumo
A síndrome dos ovários policísticos (SOP) é uma desordem endócrina complexa, frequentemente observada em mulheres na faixa de idade reprodutiva. Embora sua etiologia permaneça pouco esclarecida, há crescentes evidências de que a resistência à insulina e à hiperinsulinemia compensatória desempenha um papel central na patogênese da desordem. A resistência à insulina é intrínseca à síndrome, porém, sobrepeso, obesidade e, particularmente, obesidade central podem exacerbá-la, com possíveis consequências no fenótipo da desordem. A prevalência exata de obesidade em portadoras da síndrome dos ovários policísticos é desconhecida, sabendo-se, contudo, que o aumento de adiposidade global e visceral é bastante comum nestas pacientes e parece variar com a idade, raça, fatores genéticos e ambientais. Alguns estudos sugerem que a obesidade apresenta pouco impacto nos sinais, sintomas ou desenvolvimento da síndrome dos ovários policísticos, outros, porém, demonstram que o excesso de peso e o acúmulo de gordura intra-abdominal podem agravar a hiperandrogenemia, distúrbios menstruais, anovulação crônica, hipertensão arterial e algumas anormalidades metabólicas tais como dislipidemias e intolerância à glicose, elevando o risco de infertilidade, diabetes mellitus, aterosclerose e doença cardiovascular clínica/subclínica. A obesidade nestas pacientes também tem sido associada à redução de resposta aos tratamentos para infertilidade, elevação do risco de complicações gravídicas, câncer de endométrio e comorbidades psicológicas. A redução do peso corporal através de mudanças comportamentais, medicamentos ou cirurgia bariátrica representa, portanto, a primeira linha de abordagem terapêutica em mulheres obesas portadoras da síndrome dos ovários policísticos. Em resposta à diminuição da adiposidade, há melhora na resistência à insulina e hiperandrogenemia com benefícios em diversos parâmetros, especialmente naqueles relacionados aos distúrbios reprodutivos e metabólicos.
\end{abstract}

Descritores: Síndrome dos ovários policísticos; Resistência insulínica; Obesidade; Fenótipo; Fisiopatologia.

\begin{abstract}
Polycystic ovary syndrome (PCOS) is a complex and common endocrine disorder affecting reproductive-aged women. The etiology of polycystic ovary syndrome remains unclear but increasing evidence supports a central role of insulin resistance and its compensatory hyperinsulinemia in the pathogenesis of the syndrome. Insulin resistance is inherent to PCOS, however, it can be exacerbated by overweight, obesity and especially by central obesity, with possible consequences on the syndrome phenotype. The exact prevalence of obesity in women with polycystic ovary syndrome is not known, however, higher global adiposity and increased amounts of visceral adipose tissue are commonly observed in these patients seeming to vary with age, race, genetic and environmental factors. Although some studies suggest that obesity has little impact on symptoms, features or development of polycystic ovary syndrome, many data report that excess body weight and visceral fat accumulation may worsen certain aspects of the phenotype including biochemical hyperandrogenemia, menstrual disorders, chronic anovulation and some metabolic abnormalities such as dyslipidemia, glucose intolerance and hypertension, raising the risk for infertility, diabetes mellitus, atherosclerosis, clinical and subclinical cardiovascular disease. Obesity in women with polycystic ovary syndrome has also been associated with a poor response to infertility treatment, high risk for pregnancy complications, elevated risk for endometrial cancer and increased psychological comorbidities.

Therefore, weight loss with lifestyle therapy, pharmacological treatment and bariatric surgery is considered the first-line therapy in obese women with polycystic ovary syndrome. Weight loss improves insulin resistance and hyperandrogenism with benefits in many features, especially metabolic and reproductive abnormalities.
\end{abstract}

Keywords: Polycystic ovary syndrome; Insulin resistance; Obesity; Phenotype; Pathophysiology. 


\section{Introdução}

A síndrome dos ovários policísticos (SOP) é uma desordem heterogênea, de etiologia não estabelecida, que apresenta agregação familiar e acomete de 4 a $18 \%$ das mulheres em idade reprodutiva na dependência do critério utilizado para o diagnóstico. ${ }^{1-3}$

Segundo proposta do National Institutes of Health, o diagnóstico da SOP fundamenta-se no achado de oligo-anovulação associada ao hiperandrogenismo clínico e/ou bioquímico, após afastamento de etiologias já estabelecidas para estas condições. De acordo com o Consenso de Rotterdam, elaborado pela Sociedade de Embriologia e Reprodução Humana Europeia e pela Sociedade de Medicina Reprodutiva Americana, utilizado atualmente por cerca de $80 \%$ dos especialistas, o diagnóstico baseia-se na presença de dois dentre três dos sinais: oligo-anovulação, hiperandrogenismo clínico e/ou bioquímico e/ou morfologia ovariana policística, excluindo-se as etiologias relacionadas. ${ }^{4}$

Embora a oligo-anovulação, o hiperandrogenismo e a morfologia ovariana policística representem os sinais cardinais para o diagnóstico, observam-se frequentemente nestas pacientes importantes alterações psíquicas ${ }^{5}$ e incidência elevada de condições diretamente relacionadas à resistência à insulina (RI) tais como obesidade, dislipidemias, intolerância à glicose, diabetes mellitus tipo 2 (DM2), hipertensão arterial, síndrome metabólica, inflamação e disfunção endotelial, o que eleva significativamente o risco de doença cardiovascular neste grupo. ${ }^{6,7}$ De fato, já foi estabelecido que 25 a $50 \%$ das portadoras de SOP, independente da presença de obesidade, apresentam algum grau de RI nos tecidos muscular e adiposo relacionado a alterações intrínsecas nos receptores da insulina que incluem mutações no gene do receptor, excesso de fosforilação dos resíduos da serina, depleção de adenosina e deficiência do fator de transcrição PPAR-gama. ${ }^{8}$

$O$ excesso de peso nestas pacientes é comum e parece variar com a idade, raça e fatores ambientais. Em um estudo americano, a prevalência de obesidade elevou-se de 51 para $74 \%$, respectivamente, nos períodos de 1987-1990 e 2000-2002, acompanhando o ganho de peso da população geral, porém, descreve-se que apenas $14 \%$ das pacientes italianas e 30\% das espanholas são obesas e que, apesar do índice de massa corporal (IMC) ser significativamente maior nas mulheres com SOP quando comparado a mulheres normais, $76 \%$ das croatas e $42 \%$ das indianas com a síndrome apresentam IMC inferior a $25 \mathrm{~kg} / \mathrm{m}^{2} .^{9,10,11} \mathrm{Na}$ casuística do Hospital das Clínicas de São Paulo observa-se que $36 \%$ das portadoras de SOP são obesas, $27 \%$ têm sobrepeso e $37 \%$ apresentam IMC normal. $\mathrm{O}$ aumento da adiposidade é particularmente intraperitoneal/mesentérico, ${ }^{12}$ que pode ser detectado em adolescentes,,$^{13}$ tende a se agravar com a idade e parece ser mais acentuado em mulheres da raça negra. ${ }^{14}$

Algumas publicações sugerem que a obesidade tem discreto impacto nos sintomas ou desenvolvimento da SOP, ${ }^{9}$ enquanto outros demonstram que o excesso de peso, especialmente o acúmulo de gordura intra-abdominal, pode exacerbar algumas manifestações da desordem incluindo a ansiedade, depressão, hiperandrogenismo e particularmente as alterações menstruais, de fertilidade e distúrbios metabólicos. ${ }^{15}$

\section{Vínculo fisiopatológico entre a obesidade e a síndrome}

As inter-relações entre obesidade e SOP são complexas, porém, as evidências indicam que Rl e hiperandrogenismo representam o elo entre estas duas condições. Estudos em fêmeas demonstram que a exposição ao excesso de androgênios intra-útero, no período neonatal ou na vida adulta, aumenta a expressão de genes envolvidos na lipogênese predispondo ao acúmulo de massa gorda, particularmente na cavidade abdominal. ${ }^{16} \mathrm{O}$ depósito de gordura visceral, observado também em transexuais femininos que utilizam altas doses de testosterona e em mulheres com hiperandrogenismo endógeno, está possivelmente relacionado ao metabolismo local de esteroides, maior expressão de receptores androgênicos neste tecido e redução da lipólise no tecido celular subcutâneo induzida pelos androgênios. ${ }^{17,18}$ Este vínculo foi corroborado pela demonstração de correlação positiva entre níveis séricos de testosterona livre e adiposidade central, avaliada por densitometria, em portadoras de SOP. ${ }^{19}$

Considerando-se que a atividade lipolítica do tecido adiposo visceral em resposta às catecola- 
minas é elevada, ${ }^{18}$ postula-se que o excesso de gordura intra-abdominal forneça grande quantidade de ácidos graxos livres ao sistema porta, contribuindo para o aumento da produção de glicose pelo fígado e de insulina pelo pâncreas. ${ }^{17}$ Além disso, sabe-se que a obesidade visceral altera as concentrações de adipocinas (leptina, adiponectina, resistina, interleucina-6, fator de necrose tumoral-alfa), peptídeos que atuam como adipocinas e fatores de crescimento, com prejuízos adicionais à ação da insulina. ${ }^{20}$

É importante destacar ainda que a insulina aumenta a expressão do fator de transcrição Kruppel-like factor (KLF-15) o qual, além de estimular a adipogênese, aumenta a atividade da enzima 17-beta-hidroxiesteroide desidrogenase tipo 5, principal responsável pela conversão de androstenediona em testosterona, tanto no tecido celular subcutâneo quanto visceral. ${ }^{21}$ Adicionalmente, a insulina estimula a androgênese ovariana via aumento da secreção de hormônio luteinizante e da proteína carregadora do fator de crescimento semelhante à insulina (IGFBP-1), suprime a globulina ligadora de hormônios sexuais (sex hormone-binding globulin - SHBG), elevando a biodisponibilidade androgênica e, juntamente com a leptina, aumenta a frequência dos pulsos de hormônio liberador de gonadotrofinas favorecendo a secreção de hormônio luteinizante em detrimento do hormônio folículo estimulante. ${ }^{22}$

Portanto, embora a RI/hiperinsulinemia compensatória sejam intrínsecas à síndrome, o hiperandrogenismo estimula o ganho de peso que agrava a RI com possíveis repercussões no fenótipo da síndrome.

\section{Impacto do excesso de peso no fenótipo da síndrome}

O impacto do excesso de peso nos distúrbios metabólicos da SOP foi avaliado em recente metanálise de 30 estudos controlados. ${ }^{23}$ Pacientes obesas demonstraram maior supressão da SHBG e maiores níveis de testosterona total, índices de androgênios livres, glicose, insulina e HOMA (jejum/após sobrecarga), além de perfil lipídico mais desfavorável (maior supressão do HDL-colesterol e elevação do LDL-colesterol/triglicerídeos) quando comparadas a pacientes com IMC normal. A redução da SHBG e do HDL-colesterol e a elevação da insulina e dos triglicerídeos foi significativamente diferente mesmo nas pacientes com sobrepeso. A revisão mostrou ainda que a obesidade central associa-se a níveis mais elevados de insulina e que o hiperandrogenismo clínico nem sempre é exacerbado pelo ganho ponderal, demonstrando que a correlação entre hirsutismo e IMC na SOP parece não ser linear.

Embora mulheres com SOP possam compensar temporariamente a RI com hiperinsulinemia, verifica-se que o sobrepeso e a obesidade, assim como histórico familiar de diabetes, aceleram o processo de progressão para intolerância à glicose e DM2. ${ }^{24}$ Além disso, é importante destacar que a hipertensão arterial costuma ser observada somente em mulheres com diagnóstico de SOP obesas e que, em um grande estudo multicêntrico, a síndrome metabólica foi documentada exclusivamente nas pacientes com IMC $>27 \mathrm{~kg} /$ $\mathrm{m}^{2}{ }^{25}$ Como consenso, portanto, o excesso de peso agrava as manifestações metabólicas da SOP, acentuando o risco de diabetes e doença cardiovascular nestas pacientes.

A obesidade por si só está associada à redução de taxas ovulatórias, aumento do número de abortamentos e complicações tardias da gravidez, podendo, consequentemente, agravar o risco de infertilidade inerente à síndrome. Embora a influência do peso corporal na função ovulatória de pacientes com SOP ainda não esteja perfeitamente estabelecida, observa-se que reduções de $5 \%$ do peso corporal podem restaurar os ciclos menstruais, aumentando significativamente o número de ciclos ovulatórios. Sabe-se também que a elevação do IMC na SOP prediz tanto resistência ao clomifeno quanto falha de resposta à estimulação por gonadotrofinas em procedimentos de fertilização in vitro e que a utilização de metformina nestas pacientes, ao reduzir o peso corporal e Rl, eleva o número de gestações espontâneas e clomifeno-induzidas, ${ }^{26}$ conforme demonstram as metanálises. ${ }^{27}$

Adicionalmente, em um agrupamento sistemático de trabalhos controlados onde foram analisadas ocorrências adversas durante a gravidez, os autores concluíram que a coexistência de obesidade eleva a frequência de hipertensão e diabetes gestacional, trabalho de parto prematuro e mortalidade fetal em portadoras desta síndrome. ${ }^{28}$

Alguns estudos epidemiológicos têm sugerido também que mulheres com SOP apresentam maior risco de câncer endometrial, ${ }^{29}$ o qual estaria 
relacionado ao aumento de estrona e à elevada prevalência de ciclos anovulatórios que, sabidamente, favorecem a hiperplasia do endométrio. $\mathrm{O}$ excesso de peso, igualmente vinculado a este tipo de câncer, ${ }^{30}$ representa, portanto, um fator de risco adicional para a neoplasia de endométrio nestas pacientes.

Por fim, importa salientar que ansiedade, depressão, estresse e insatisfação pessoal, relatados frequentemente por mulheres acometidas pela SOP, poderiam ser agravados pela alteração da imagem corporal consequente ao ganho de peso. Até o momento, entretanto, a contribuição da obesidade nos parâmetros psicológicos destas pacientes não foi satisfatoriamente documentada. ${ }^{5}$

\section{Conclusão}

A obesidade apresenta um efeito independente e aditivo nos fatores de risco metabólicos e reprodutivos da SOP. Este efeito está relacionado ao agravamento da $\mathrm{RI}$, intrínseca à síndrome. $\mathrm{O}$ controle do ganho ponderal através de medidas comportamentais, medicamentos ou cirurgia bariátrica representa possivelmente o principal parâmetro modificável na abordagem da fertilidade e na prevenção de complicações cardiovasculares neste grupo.

\section{Referências}

1. Ehrmann DA. Polycystic ovary syndrome. N Engl J Med. 2005 Mar;352(12):1223-36.

2. Azziz R, Woods KS, Reyna R, Key TJ, Knochenhauer ES, Yildiz BO. The Prevalence and Features of the Polycystic Ovary Syndrome in an Unselected Population. J Clin Endocrinol Metab. 2004 Jun;89(6):2745-9.

3. March WA, Moore VM, Willson KJ, Phillips DI, Norman RJ, Davies MJ. The prevalence of polycystic ovary syndrome in a community sample assessed under contrasting diagnostic criteria. Human Reprod. 2010 Feb;25(2):544-51. Epub 2009 Nov 12. http://dx. doi.org/10.1093/humrep/dep399

4. Rotterdam ESHRE/ASRM-Sponsored PCOS Consensus Workshop Group. Revised 2003 consensus on diagnostic criteria and long term health risks related to polycystic ovary syndrome. Fertil Steril. 2004 Jan;81(1):19-25.

5. Li Y, Yu Ng EH, Stener-Victorin E, Hou L, Wu T, Han F, et al. Polycystic ovary syndrome is associated with negatively variable impacts on domains of health-related quality of life: evidence from a meta-analysis.
Fertil Steril. 2011 Aug;96(2):452-8. http://dx.doi. org/10.1016/j.fertnstert.2011.05.072

6. Moran LJ, Misso ML, Wild RA, Norman RJ. Impaired glucose tolerance, type 2 diabetes and metabolic syndrome in polycystic ovary syndrome: a systematic review and meta-analysis. Human Reprod Update. 2010 Jul-Aug;16(4):347-63. Epub 2010 Feb 16. http:// dx.doi.org/10.1093/humupd/dmq001

7. Teede H, Deeks A, Moran L. Polycystic ovary syndrome: a complex condition with psychological, reproductive and metabolic manifestations that impacts on health across the lifespan. BMC Med. 2010 Jun;30:8-41. http://dx.doi.org/10.1186/1741-7015-8-41

8. Meyer C, McGrath BP, Teede HJ. Overweight Women with Polycystic Ovary Syndrome have evidence of subclinical cardiovascular disease. J Clin Endocrinol Metab. 2005 Oct;90(10):5711-6.

9. Yildiz BO, Knochenhauer ES, Azziz R. Impact of obesity on the risk for polycystic ovary syndrome. J Clin Endocrinol Metab. 2008 Jan;93(1):162-8.

10. Baldani DP, Skrgatić L, Goldstajn MS, Zlopasa G, Oguić SK, Canić T, et al. Clinical and biochemical characteristics of polycystic ovary syndrome in Croatian population. Coll Antropol. 2012 Dec;36(4):14138.

11. Saxena P, Prakash A, Nigam A, Mishra A. Polycystic ovary syndrome: Is obesity a sine qua non? A clinical, hormonal, and metabolic assessment in relation to body mass index. Indian J Endocrinol Metab. 2012 Nov;16(6):996-9. http://dx.doi.org/10.4103/22308210.103011

12. Borruel S, Fernández-Durán E, Alpañés M, Martí D, Alvarez-Blasco F, Luque-Ramirez M, et al. Global adiposity and thickness of intraperitoneal and mesenteric adipose tissue depots are increased in women with polycystic ovary syndrome (PCOS). J Clin Endocrinol Metab. 2013 Mar;98(3):1254-63. http://dx.doi.org/10.1210/jc.2012-3698

13. Nair MK, Pappachan P, Balakrishnan S, Leena ML, George B, Russell PS. Menstrual irregularity and polycystic ovarian syndrome among adolescent girls--a 2 year follow-up study. Indian J Pediatr. 2012 Jan;79 Suppl 1:S69-73. http://dx.doi.org/10.1007/s12098-0110432-y

14. Glueck CJ, Morrison JA, Daniels S, Wang P, Stroop D. Sex hormone-binding globulin, oligomenorrhea, polycystic ovary syndrome, and childhood insulin at age 14 years predict metabolic syndrome and class III obesity at age 24 years. J Pediatr. 2011 Aug;159(2):308-13. http://dx.doi.org/10.1016/j. jpeds.2011.01.018

15. Liou TH, Yang JH, Hsieh CH, Lee CY, Hsu CS, Hsu MI. Clinical and biochemical presentations of polycystic ovary syndrome among obese and nonobese women. Fertil Steril. 2009;92(6):1960-5. http://dx.doi. 


\section{org/10.1016/j.fertnstert.2008.09.003}

16. Swinnen JV, Heemers H, van de Sande T, de Schrijver E, Brusselmans K, Heyns W, et al. Androgens, lipogenesis and prostate cancer. J Steroid Biochem Mol Biol. 2004 Nov;92(4):273-9.

17. Blouin K, Veilleux A, Luu-The V, Tchernof A. Androgen metabolism in adipose tissue: recent advances. Mol Cell Endocrinol. 2009 Mar 25;301(1-2):97-103. http://dx.doi.org/10.1016/j.mce.2008.10.035

18. Dicker A, Rydén M, Näslund E, Muehlen IE, Wirén M, Lafontan M, et al. Effect of testosterone on lipolysis in human pré-adipocytes from different fat depots. Diabetologia. 2004 Mar;47(3):420-8.

19. Yucel A, Noyan V, Sagsoz N. The association of serum androgens and insulin resistance with fat distribution in polycystic ovary syndrome. Eur J Obstet Gynecol Reprod Biol. 2006 May 1;126(1):81-6.

20. Yildiz BO, Azziz R, Androgen Excess and PCOS Society. Ovarian and adipose tissue dysfunction in polycystic ovary syndrome: report of the 4th special scientific meeting of the Androgen Excess and PCOS Society. Fertil Steril. 2010 Jul;94(2):690-3. http://dx. doi.org/10.1016/j.fertnstert.2009.03.058

21. Du X, Rosenfield RL, Qin K. KLF 15 is a transcriptional regulator of human 17 beta-hidroxysteroid dehydrogenase type 5 gene. A potencial link between regulation of testosterone production and fat stores in women. J Clin Endocrinol Metab. 2009 Jul;94(7):2594-601. http://dx.doi.org/10.1210/jc.20090139

22. Legro RS. Obesity and PCOS: implications for diagnosis and treatment. Semin Reprod Med. 2012 Dec;30(6):496-506. http://dx.doi. org/10.1055/s-0032-1328878

23. Lim SS, Norman RJ, Davies MJ, Moran LJ. The effect of obesity on polycystic ovary syndrome: a systematic review and meta-analysis. Obes Rev. 2013 Feb;14(2):95-109. http://dx.doi.org/10.1111/j.1467789X.2012.01053.x

24. Wang JX, Norman RJ. Risk factors for the deterioration of glucose metabolism in polycystic ovary syndrome. Reprod Biomed Online. 2004 Aug;9(2):201-4.

25. Ehrmann DA, Liljenquist DR, Kasza K, Azziz R, Legro RS, Ghazzi MN, et al. Prevalence and predictors of metabolic syndrome in women with polycystic ovary syndrome. J Clin Endocrinol Metab. 2006 Jan;91(1):48-53.

26. Rausch ME, Legro RS, Barnhart HX, Schlaff WD, Carr BR, Diamond MP, et al. Predictors of pregnancy in women with polycystic ovary syndrome. J Clin Endocrinol Metab. 2009 Sep;94(9):3458-66. http:// dx.doi.org/10.1210/jc.2009-0545

27. Sun X, Zhang D, Zhang W. Effect of metformin on ovulation and reproductive outcomes in women with polycystic ovary syndrome: a meta-analysis of randomized controlled trials. Arch Gynecol Obstet. 2013 Aug;288(2):423-30. http://dx.doi.org/10.1007/ s00404-013-2756-5.

28. Boomsma CM, Eijkemans MJ, Hughes EG, Visser GH, Fauser BC, Macklon NS. A meta-analysis of pregnancy outcomes in women with polycystic ovary syndrome. Hum Reprod Update. 2006 Dec;12(6):67383.

29. Schmeler KM, Soliman PT, Sun CC, Slomovitz BM, Gershenson DM, Lu KH. Endometrial cancer in young, normal-weight women. Gynecol Oncol. 2005 Nov;99(2):388-92.

30. Kaaks R, Lukanova A, Kurzer MS. Obesity, endogenous hormones, and endometrial cancer risk: a synthetic review. Cancer Epidemiol Biomarkers Prev. 2002 Dec;11(12):1531-43.

Recebido: $\quad$ 19/08/2013.

Revisado: $\quad$ 26/11/2013.

Aprovado: $\quad$ 27/01/2014. 